\title{
Adaptive Technique for Image Fusion based on Non-Subsampled Shearlet Transform-Spatial Frequency-Human Visual Factor
}

\author{
NIU Ling and FENG Gao-feng \\ Zhou Kou Normal University, Zhoukou 466001, China, \\ JiYuan Vocational And Technical College, JiYuan Henan 454650, China \\ fengjyjava@126.com,Niuling@zknu.edu.cn
}

\begin{abstract}
As a novel multi-scale geometrical analysis theory, non-subsampled shearlet transform (NSST) own much better competences of image processing. An adaptive technique for image fusion based on NSST-spatial frequency (SF)-human visual factor $(H V F)$ is proposed in this paper. Each source image can be converted into corresponding multi-scale and multi-directional frameworks via NSST. SF and HVF are utilized to conduct the fusion courses of low-frequency and high-frequency sub images, respectively. Besides, an adaptive fusion algorithm based on NSST is devised. Finally, the final fused image can be obtained by using inverse NSST. Simulation experimental demonstrates that, compared with other classic techniques, the proposed technique has much better performance in terms of visual performance and information capturing.
\end{abstract}

Keywords: non-subsampled shearlet transform, spatial frequency, human visual factor, adaptive

\section{Introduction}

Image fusion theory is an important branch and an interested research point in the field of information fusion. At present, image fusion theory has a widespread application of medical science[1,2], remote sensing[3] and so on. The research on the kinds of techniques and technologies for image fusion can be trace back to 80's of the last century. Nowadays, the above field has been attached more and more importance by many civil and abroad scholars. Currently, many kinds of techniques for image fusion have come into being.

Among above, it is general that former pixel matrix in the original images are just deal for space domain technique. Then effective selection for original images' pixel has been realized by the way of being average and weighted. Finally, a new images' pixel matrix has been recomposed of the selected pixels. The advantages of space domain technique are simple thought process and low computing complexity. And the disadvantage is that their image fusion results are commonly not very well. Technique for transform domain is that original images decompose a group of microcosmic sub-image according to multiscale and multi-directions. In detail, the specific steps referred contain resolution, microcosmic fusion and integrated consolidation. Furthermore, microcosmic sub-images fusion is achieved following with corresponding scale and directions, and obtain the ending images fusion by inverse transformation of microcosmic sub-images.

In many kinds of transform domain techniques, classical methods contain Discrete Wavelet Transform[4], Contourlet Transform[5,6] and Non-Subsampled Contourlet Transform (NSCT) [7,8] and so on. Among above, the applications of NSCT fusion theory appear to be very widespread. Comparing to discrete wavelet transform and contourlet transform, the superiorities of NSCT are translational invariance and the performance of catching images' information. However, much too high-cost computing complexity has been restricted its practical application fields, resulting to the bottleneck 
of NSCT theory development come into being. Above all, Easley proposed a novel model called Non-Subsampled Shearlet Transform (NSST) [9]. Not only NSST has the advanced performance of catching images' characters, but also the novel model has a low computing complexity. Besides, NSST theory can effectively overcome NSCT theory's disadvantage that has a not good real time performance.

In this paper we propose an adaptive technique for image fusion based on NSST, Spatial frequency (SF) and Human Visual Factor (HVF). First of all, we take full advantage of NSST model's superior performance of catching images' information. In additional, we regard SF and HVF as sub-images fusion basis, aiming at realizing the fusion process of low-frequency and high-frequency microcosmic sub-images.

\section{NSST Basic Model}

As the dimension $n=2$, the affined coefficient formula of fusion and expansion is as follows.

$M_{A B}(\psi)=\left\{\psi_{i, j, k}(x)=|\operatorname{det} A|^{i / 2} \psi\left(B^{j} A^{i} x-k\right): x, y \in Z, k \in Z^{2}\right\}$

In the above formula, $\psi \in L^{2}\left(R^{2}\right), A$ and $B$ are both $2 \times 2$ invertible matrix, $|\operatorname{det} B|=1$. If $M_{A B}(\psi)$ is satisfied with tight frame conditions, then the elements of $M_{A B}(\psi)$ is regarded as wavelet synthesis. $A$ is an anisotropy expansion matrix, and $A^{i}$ is related to scale transformation. $B$ is a shear matrix, and $B^{j}$ is also a shear matrix that is related to geometric transformation for keeping area constant. The formulas of $A$ and $B$ are respectively regarded as $A=\left[a 0,0 a^{1 / 2}\right], B=\left[\begin{array}{lll}1 & s, 0 & 1\end{array}\right]$. In general, $a=4, s=1$, that is be $A=[4$ $0,02], B=\left[\begin{array}{lll}1 & 1,0 & 1\end{array}\right]$. Thus the above wavelet is shearlet.

Essentially, NSST is a redundancy transform, decomposition progress of that can be depart into two parts, including multi-scale and multi-direction. Multi-scale decomposition has also adopted the way of non-subsampled pyramid. Original images can be obtained a low-frequency subband image and a high-frequency subband image by decomposing at each grade. Next every grades' decompositions is dealt just as for lowfrequency subband images. After $k$ grades' multi-scale decomposition, each original image can also be come into being only one low-frequency subband image and $k$ highfrequency subband images, whose dimension and size are consistent with the original images'. Obviously, as for the progress of multi-scale decomposition are consistent. Different from NSCT, the multi-direction decomposition of NSST is that normal shearlet wave filter is mapped from pseudo polarization network system to Cartesian coordinate system. Because of abandoning subsample progress, translation inflexibility is realized, effectively, the ringing effects of discrete wavelet transform and contourlet transform are prevented. If NSST some scale subband image is decomposed at $l$ grade non-subsample sheartlet direction based on NSST, obtaining $2^{l}$ subband images consistent with original images' size and direction. Thus the above progress can realize the more accurate direction decomposition of frequency domain. In picture 1, a NSST decomposition instance of Zoneplate image is given. And the scale decomposition quantity is set to be 2, each grade of that set to be 4 .
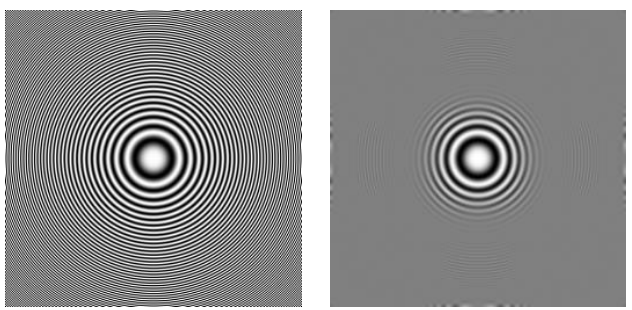

(a) Original image (b) Low-frequency image 

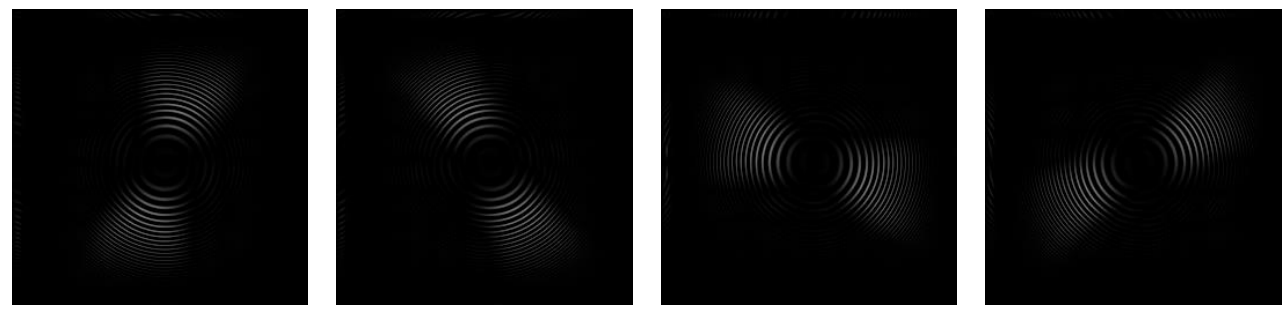

(c) 4 directions of I scale localization images
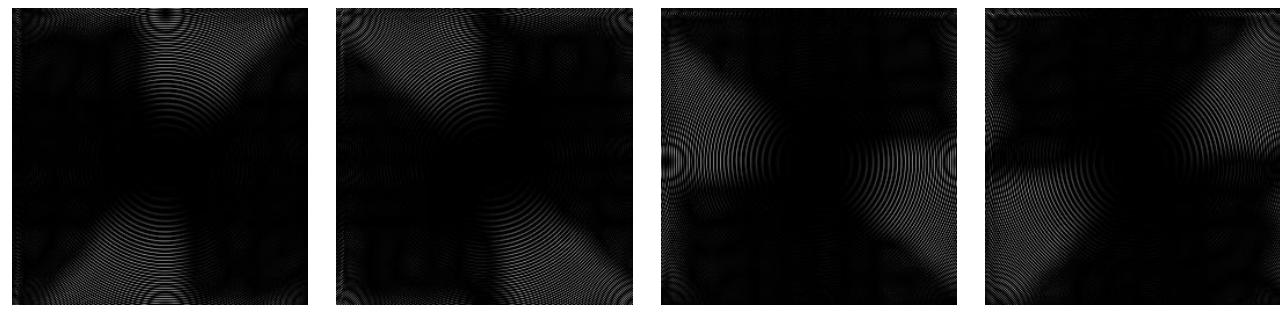

(d) 4 directions of II scale localization images

Figure 1. Non-Subsample Sheartlet Transform Decomposition Instance

\section{Image adaptive fusion frame based on NSST}

\subsection{Low-Frequency Microcosmic Subband Image Fusion}

After the decomposition of NSST, each original image can be decomposed into a series of microcosmic sub-images, that include only one low-frequency microcosmic subimage and some high-frequency microcosmic sub-images. And low-frequency microcosmic sub-images contain the most and main part information. Traditional and classical algorithm is commonly selected to add and average some low-frequency subband images' matrix. Thus the above algorithm seems to be simple. But the images' contrast degree must be reduced, additionally, each low-frequency subband image's pixel distribution are lack of consideration in the algorithm.

It is obviously appeared the differences between the pixel gray values from important targets information and surrounding pixel points' information in images. Images' space spatial frequency $(S F)^{[11]}$ can be reflected pixel points' active index. The more is the $S F$ value, the more active is the images' pixel points.

Therefore, we select $S F$ to be basis for realizing fusion progress of low-frequency microcosmic sub-image. $S F$ 's formula is as follows.

$S F=\sqrt{(R F)^{2}+(C F)^{2}+(M D F)^{2}+(S D F)^{2}}$

As for the image $I(i, j)$, the series of formulas are as follows, including $R F, C F, M D F$ and $S D F$.

$$
\begin{aligned}
& R F=\sqrt{\frac{1}{M N} \sum_{i=1}^{M} \sum_{j=2}^{N}[I(i, j)-I(i, j-1)]^{2}} \\
& C F=\sqrt{\frac{1}{M N} \sum_{j=1}^{N} \sum_{i=2}^{M}[I(i, j)-I(i-1, j)]^{2}} \\
& M D F=\sqrt{\omega_{d} \frac{1}{M N} \sum_{i=2}^{M} \sum_{j=2}^{N}[I(i, j)-I(i-1, j-1)]^{2}} \\
& S D F=\sqrt{\omega_{d} \frac{1}{M N} \sum_{j=1}^{N-1} \sum_{i=2}^{M}[I(i, j)-I(i-1, j+1)]^{2}}
\end{aligned}
$$


In the above formulas, $R F, C F, M D F$ and $S D F$ are respectively represented as 4 directions' first order gradient value, containing horizontal direction, vertical direction, the main diagonal direction and vice diagonal direction. $\omega \mathrm{d}$ is represented as distance weight value, and set here $\omega \mathrm{d}=1 / 2^{1 / 2}$.

Obviously, according to the formula (3)-(6), we can compute the images' each pixel point SF value. When it comes to the bigger SF values, their corresponding pixel points at 4 directions are more active and outstanding, that is to say, the information of which are ampler and richer.

\subsection{High-Frequency Microcosmic Subband Image Fusion}

Different from low-frequency microcosmic sub-images, high-frequency microcosmic sub-images stress on description of trivial information in original images, such as targets' borderline and so on. The disadvantage of traditional weighted average fusion algorithm can also cause contrast level of image fusion descended. It is beneficial for absolute value method to keep higher lightness' border detailed information. However, because some absolute value of pixel points' grey level at lower lightness' border are less, the structure can be easily soften, resulting in lack of borders' information.

Recently, the research on Human Visual Factor (HVF) is done more and more common concern by civil and national experts and scholars. Additionally, the research development emphasis on image fusion is converted from previous mechanical weighted model to biology theory model. The information of high-frequency subband images are generally inferior to high-frequency's. Thus we have selected HVF to be fusion basis for carrying out high-frequency subband information fusion, owing to HVF's biology theory background.

HVF can use sensitive reflection mechanism of biological vision to separate the images two parts containing target and background, that is:

$I=O+B$

Where $I, O$ and $B$ denote the original image, target information and background information, respectively.

In the practical analysis progress, high-frequency microcosmic information is considered as target information $O$, and low-frequency microcosmic information is considered as background information $B$. The gray values of high-frequency and corresponding low-frequency subband information are all bigger, which indicates the expressing information at pixel point is more plentiful. The descriptive formula is as follows.

$H V F=\frac{\left|I^{S, L}(i, j)\right|}{I^{S-1}(i, j)^{*}}$

Where HVF is human visual factor, $\|$ is absolute value symbol. $I^{S, L}(i, j)$ is highfrequency subband image coefficient whose decomposition scale and direction are $S$ and $L . I^{S-1}(i, j)^{*}$ is average coefficient value of low-frequency microcosmic subband image with $S$-1 decomposition scale. When $S=1, I^{S-1}(i, j)^{*}$ denotes area coefficient average value of original images, the formula of $I^{S-1}(i, j)^{*}$ is:

$I^{S-1}(i, j)^{*}=\frac{1}{M \times N} \sum_{x=-\frac{M-1}{2}}^{\frac{M-1}{2}} \sum_{y=-\frac{N-1}{2}}^{\frac{N-1}{2}} I^{S-1}(i+x, j+y)$

\section{Proposed Technique}

In this paper, we first use NSST theory to obtain a series of microcosmic subband images with different scale and direction. Then SF and HVF are effectively selected for 
realizing image fusion progress of corresponding scale and direction. Ultimately, we can get the ending results because of NSST inverse transformation. The entire image fusion progress is showed in Figure 2.

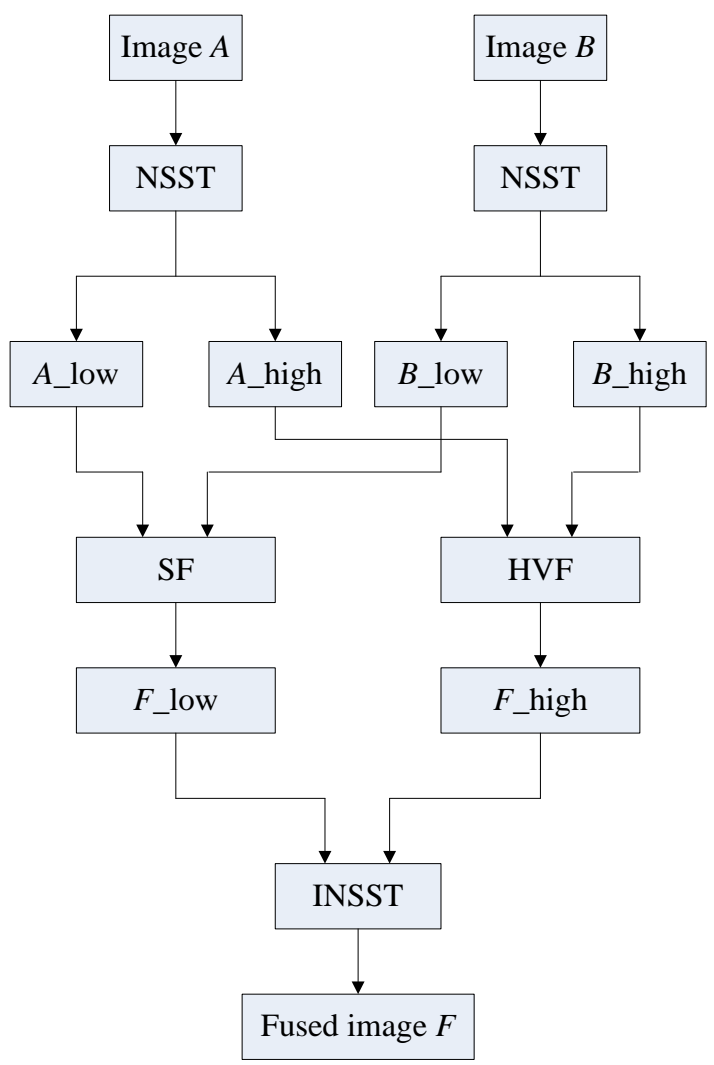

Figure 2. Image Fusion Progress based on NSST

Where $A, B$ are two original images, low and high denote respectively low-frequency subband image and high-frequency subband image. INSST is an inverse transformation of NSST. Adaptive technique for image fusion based on NSST-SF-HVF is as follows.

INPUT: Two original images $A$ and $B$;

OUTPUT: The fused image $F$;

\section{STEPS :}

1. Original images are decomposed to low-frequency microcosmic coefficient $\left\{A^{S, 0}\right.$, $\left.B^{S, 0}\right\}$ and high-frequency microcosmic coefficient $\left\{A^{S, L}, B^{S, L}\right\}$ based on NSST. Where scale grades $S$ and $L$ are the decomposition grade at $S$ direction.

2. Make use of $S F$ to accomplish fusion progress of low-frequency microcosmic subband images:

$F_{-}$low $^{S, 0}=\left\{\begin{array}{lll}A^{S, 0}, & \text { if } & S F_{A}(i, j)>S F_{B}(i, j) \\ B^{S, 0}, & \text { if } & S F_{A}(i, j)<S F_{B}(i, j) \\ \frac{A^{S, 0}+B^{S, 0}}{2}, & \text { if } & S F_{A}(i, j)=S F_{B}(i, j)\end{array}\right.$

3. Use $H V F$ to select the high-frequency microcosmic subband coefficient from original images $A$ and $B$, the corresponding formula is showed in formula (11). 


$$
F_{-} h_{i g h} h^{S, L}=\left\{\begin{array}{l}
A^{S, L}, \quad \text { if } \quad \operatorname{HVF}_{A}(i, j)>S F_{B}(i, j) \\
B^{S, L}, \quad \text { if } \quad \operatorname{HVF}_{A}(i, j)<S F_{B}(i, j) \\
A^{S, L}, \text { if } \quad H V F_{A}(i, j)=S F_{B}(i, j) \& \&\left\|A^{S, L}\right\|>\left\|B^{S, L}\right\| \\
B^{S, L}, \text { if } \quad H V F_{A}(i, j)=S F_{B}(i, j) \& \&\left\|A^{S, L}\right\|<\left\|B^{S, L}\right\| \\
\frac{A^{S, L}+B^{S, L}}{2}, \text { else }
\end{array}\right.
$$

4. Utilize NSST inverse transformation as to low-frequency microcosmic subband images on after-fusion and a series of high-frequency microcosmic subband images to get the ending image $F$.

\section{Experimental Results and Analysis}

For verifying the practical performance of the proposed technique, we utilize the MATLAB software to emulate, compare and analyze the fusion results of several compared fusion techniques and the proposed technique.

\subsection{Experimental Condition}

Simulation experiments are carried out in a PC with Windows $7 / 2.1 \mathrm{GHz} / 2 \mathrm{G}$. In this section, we select different kinds of original images with three imaging mechanisms to carry out the experiments, which are referred to multi-focus Clock images, multispectral SAR images and visible light quad and infrared quad images. And three pairs of original images' size are all $512 \times 512$. Moreover, we choose three techniques comparing with the novel technique, including the coupling of multi-channel pulse neural network technique (technique 1) ${ }^{[12]}$, shearlet transform technique (technique 2) ${ }^{[13]}$ and Laplace transform (technique 3$)^{[14]}$. In order to assure the objectivity of fusion results, all the parameters in technique 1, 2 and 3 are also the existing set mode's values in references.

Furthermore, the performance evaluation' ways as to the ending fusion results are subjective visible evaluation and objective index values evaluation. And Information Entropy (IE) ${ }^{[15]}$, Mutual Information (MI) ${ }^{[15]}$ are chosen to be evaluation index. The index value of IE denotes the quantity of information in image. And the bigger are the values, the information in fusion images are ampler. The index value of MI denotes the mutual information between the original images and the ending fusion images. The value of MI is bigger, the original information are ampler. The index value of SF denotes the activity degree of each pixel point to some extent. That is to say the bigger value of SF represents the ampler obvious information in fusion images. Besides, the concrete details on IE, MI, SF can use the references $[11,15]$ for reference.

\subsection{Experimental Results and Analysis}

Three groups of original images simulation are respectively showed in Figure 3. The first group images are multi-focus images. In Figure 3(a), the right side and the left side of the alarm clocks are respectively in the focus area and out-of-focus area. In Figure 3(b), it is yet an inverse circumstance to the above distribution in Figure 3(a). Hence it is necessary to inject two original images' focus area information extracted. Figure 3(c) and Figure 3(d) are an identical SAR figure with different wave bands and their own specific information. It is no doubt that the visible results can be greatly improved just by realizing the effective fusion. The last group images simulation are grey visible light and infrared images. The grey visible light images can just descript the main information in the visible light with grey image sensor, yet not expressing target information in the complex background. 
In contrast, infrared images generally focus on catching and expressing heat source information, which is beneficial to capture the infrared information in the complex background circumstance. Therefore, how to effectively fuse the information of both background and infrared heat source must be a significant problem which is also worthy of research. Thus all the simulation experiments are carried out based on the proposed technique and three other compared techniques, whose simulation results are showed in Figure 4-Figure 6. Obviously, the result images based on the proposed technique have distinct superiorities over those based on other three ones.

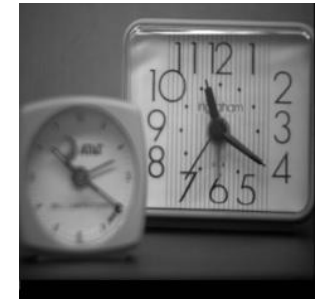

(a) Right focus image

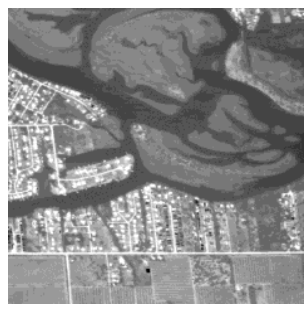

(d) 3 wave bands SAR image

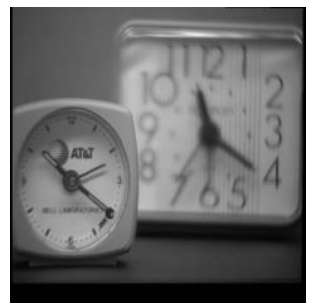

(b) Left focus image

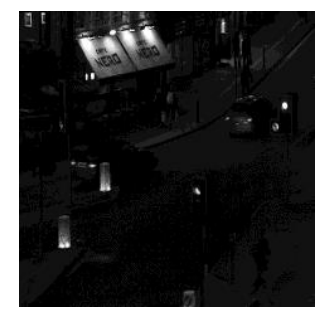

(e) Visible image

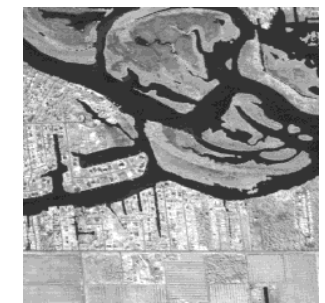

(c) 8 wave bands SAR image

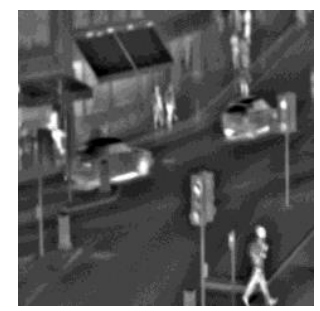

(f) Infrared image

Figure 3. Original Images Simulation
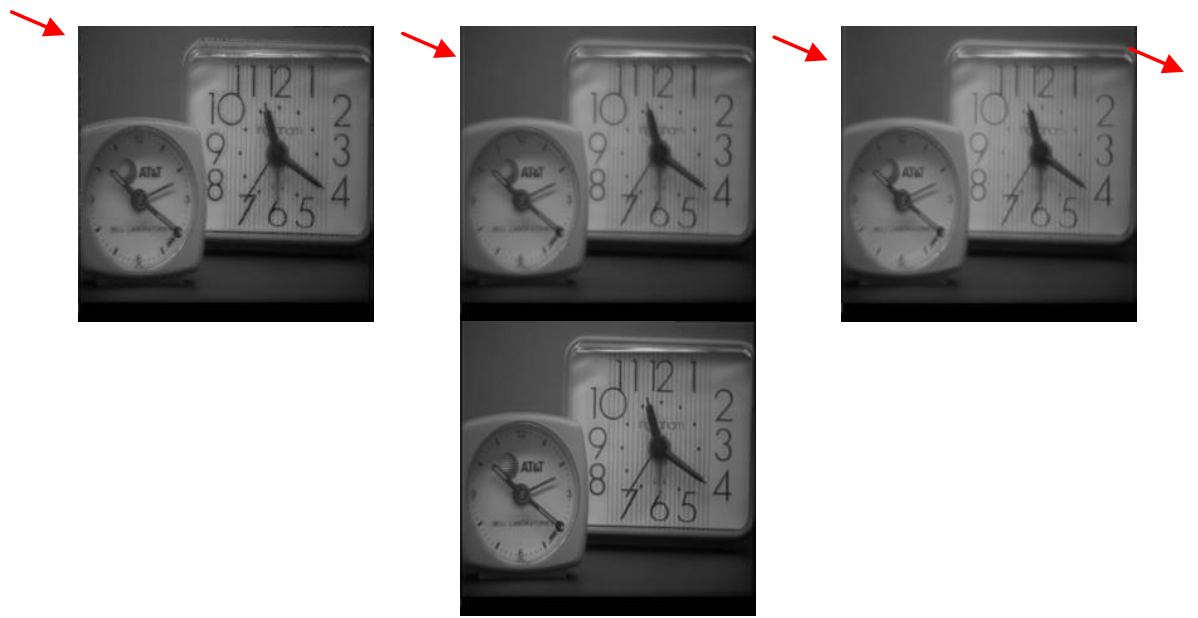

(a)fusion results of

(b) fusion results of fusion results of

technique 1

technique 2

technique 3

(c) fusion results of

(d) technique

Figure 4. Multi-Focus Images' Fusion Results 

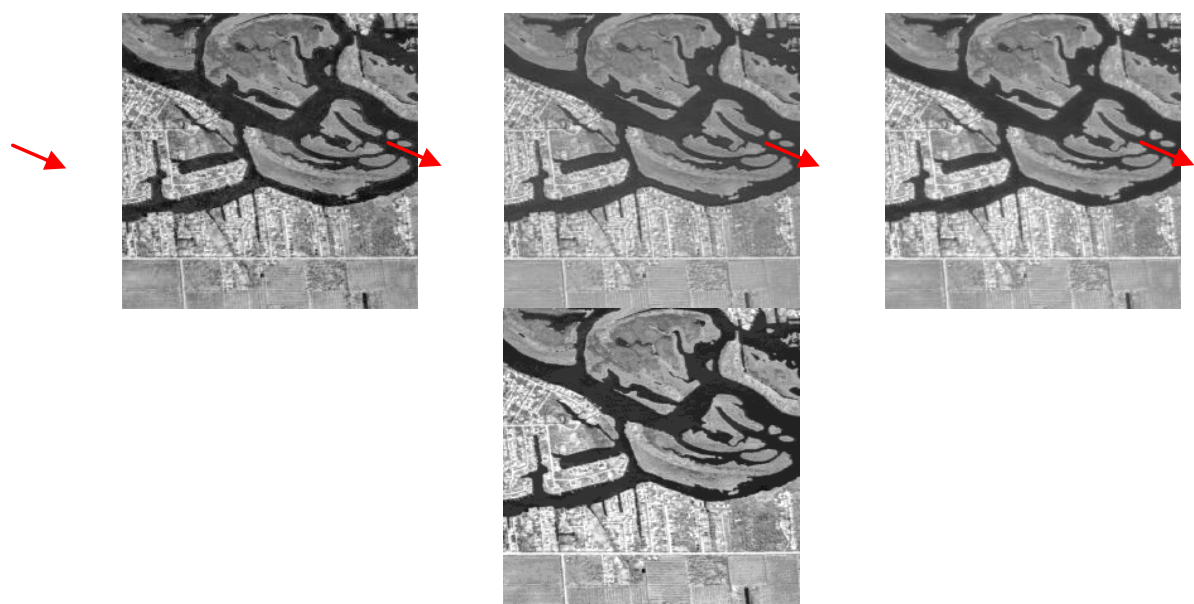

(a)fusion results of

(b) fusion results of fusion results of

technique 1

technique 2

technique

technique 3

the proposed

Figure 5. Multispectral SAR Images' Fusion Results
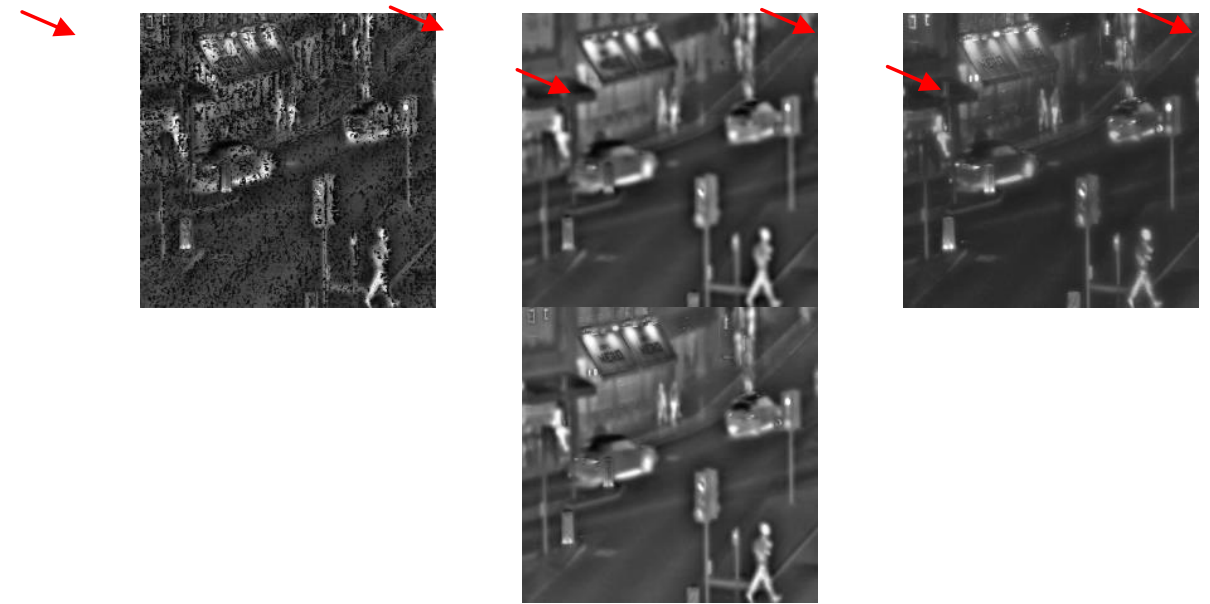

(d)
(c) fusion results of 


\section{Acknowledgements}

The authors thank the anonymous reviewers and editors for their invaluable suggestions.

\section{References}

[1] Y. C. Yang, J. W. Dang and Y. P. Wang, "A Medical Image Fusion Method Based on Lifting Wavelet Transform and Adaptive PCNN", Journal of Computer-aided Design \& Computer Graphics, vol. 24, no. 4, (2004), pp. 494-499.

[2] Z. P. Xu. "Medical image fusion using multi-level local extrema", Information Fusion, vol. 19, no. 1, (2014), pp. 38-48

[3] W. Q. Wang, L. C. Jiao and S. Y. Yang, "Fusion of multispectral and panchromatic images via sparse representation and local autoregressive model”, Information Fusion, vol. 20, no. 1, (2014), pp. 73-87.

[4] J. Tian and L. Chen, "Adaptive multi-focus image fusion using a wavelet-based statistical sharpness measure”, Signal Processing, vol. 92, no. 9, (2012), pp. 2137-2146.

[5] Y. A. Zheng, J. S. Song, W. M. Zhou and R. H. Wang, "False Color Fusion for Multi-band SAR Images Based on Contourlet Transform", Acta Automatica Sinica, vol. 33, no. 4, (2007), pp. 337-341.

[6] G. S. Hu, D. Liao and J. Kong, "Remote Sensing Image Fusion Based on SupportVector Value Contourlet Transform", Acta Electronica Sinica, vol. 38, no. 6, (2010), pp. 1287-1292.

[7] J. H. Adu, J. H. Gan, Y. Wang and J. Huang, "Image fusion based on nonsubsampled contourlet transform for infrared and visible light image", Infrared Physics \& Technology, vol. 61, no. 1, (2013), pp. 94-100.

[8] T. H. Chen and Y. C. Lin, "Infrared and visible image fusion method based on developed NSCT transform”, Journal of Beijing Jiaotong University, vol. 37, no. 6, (2013), pp. 18-21.

[9] G. Easley, D. Labate and W. Q. Lim. Sparse directional image representation using the discrete shearlet transforms, Applied and Computational Harmonic Analysis, vol. 25, no. 1, (2008), pp. 25-46.

[10] W. W. Kong and Y. J. Lei, "Technique for Image Fusion Based on Non-Subsampled Shearlet Transform and Human Visual Characteristic", Journal of Harbin Engineering University, vol. 34, no. 6, (2013), pp. 777-782.

[11] Y. Zheng, E. A. Essock, B. C. Hansen and A. M. Haun, "A new metric based on extended spatial frequency and its application to DWT based fusion algorithms", Information Fusion, vol. 8, no. 2, (2007), pp. 177-192.

[12] Z. B. Wang and Y. D. Ma, "Medical image fusion using m-PCNN", Information. Fusion, vol. 9, no. 2, (2008), pp. 176-185.

[13] Q. G. Miao, C. Shi, P. F. Xu, M. Yang and Y. B. Shi, "A novel algorithm of image fusion using shearlets", Optics Communication, vol. 284, no. 6, (2011), pp. 1540-1547.

[14] P. Burt and E. Adelson. The laplacian pyramid as a compact image code, IEEE Transactions on Communications, vol. 31, no. 4, (1983), pp. 532-540.

[15] Z. Liu, E. Blasch, Z. Y. Xue, J. Y. Zhao, R. Laganiere and W. Wu, "Fusion algorithms for context enhancement in night vision: a comparative study", IEEE Transactions on Pattern Analysis and Machine Intelligence, vol. 34, 1, (2012), pp. 94-109.

Author

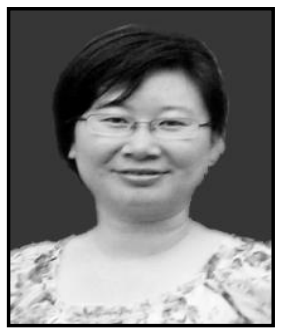

Niu Ling, She received the B.Eng degree in Computer science from Henan normal university and M.Eng degree in Computer science from Chengdu University of Technology. She is currently researching on computer application technology.

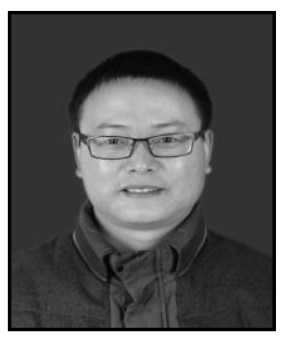

Feng Gao-feng, He received the computer science degree from Henan Normal University, China, and the master degree in computer science from Beijing University of Posts and Telecommunications, $\mathrm{He}$ is a member of China Computer Federation and Association of Fundamental Computing Education in Chinese Universities in Beijing, China. 
International Journal of Signal Processing, Image Processing and Pattern Recognition Vol.9, No.3 (2016) 